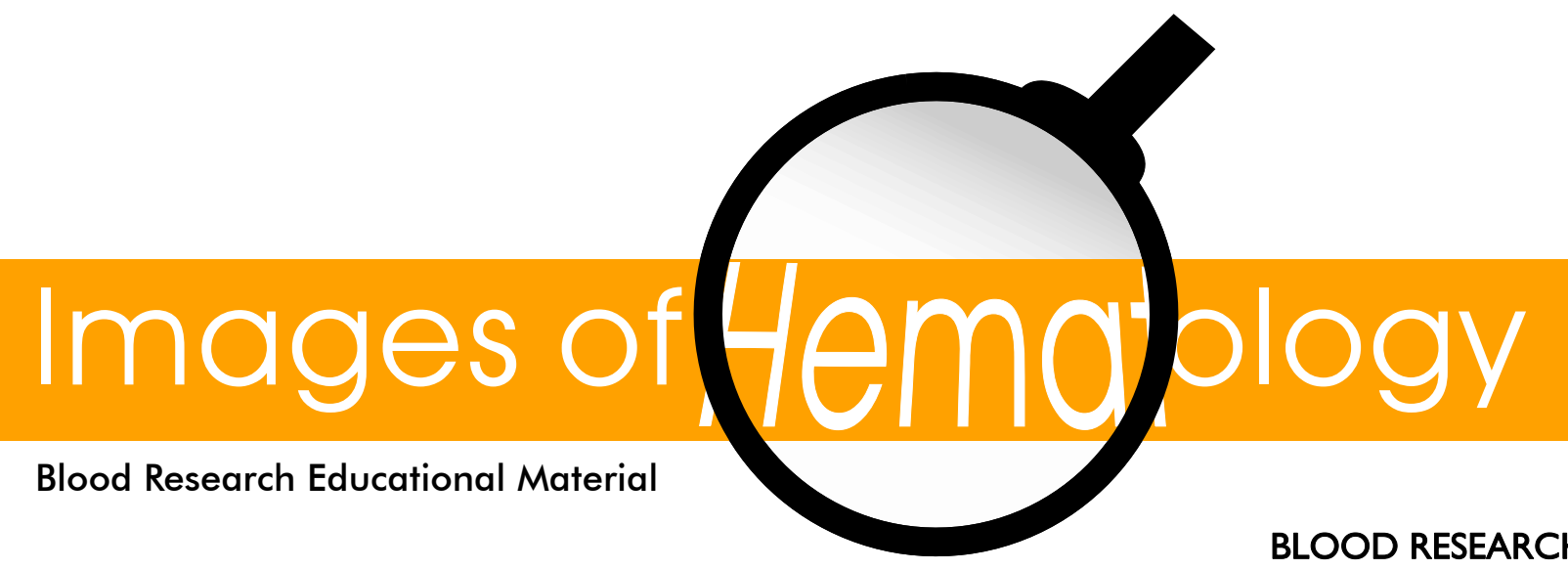

Volume $55 \cdot$ Number $4 \cdot$ December 2020 https://doi.org/10.5045/br.2020.2020156

\title{
Bone marrow involvement by primary oxalosis
}

Stefano Fratoni ${ }^{1}$, Pasquale Niscola ${ }^{2}$

${ }^{1}$ Surgical Pathology, Hematopathology Unit, ${ }^{2}$ Haematology Unit, St. Eugenio Hospital, Rome, Italy

Received on June 29, 2020; Revised on October 23, 2020; Accepted on November 12, 2020

Correspondence to Pasquale Niscola, M.D., Hematology Unit, St. Eugenio Hospital, ASL Roma 2, Piazzale dell’Umanesimo 10, Rome 00144, Italy E-mail: pniscola@gmail.com
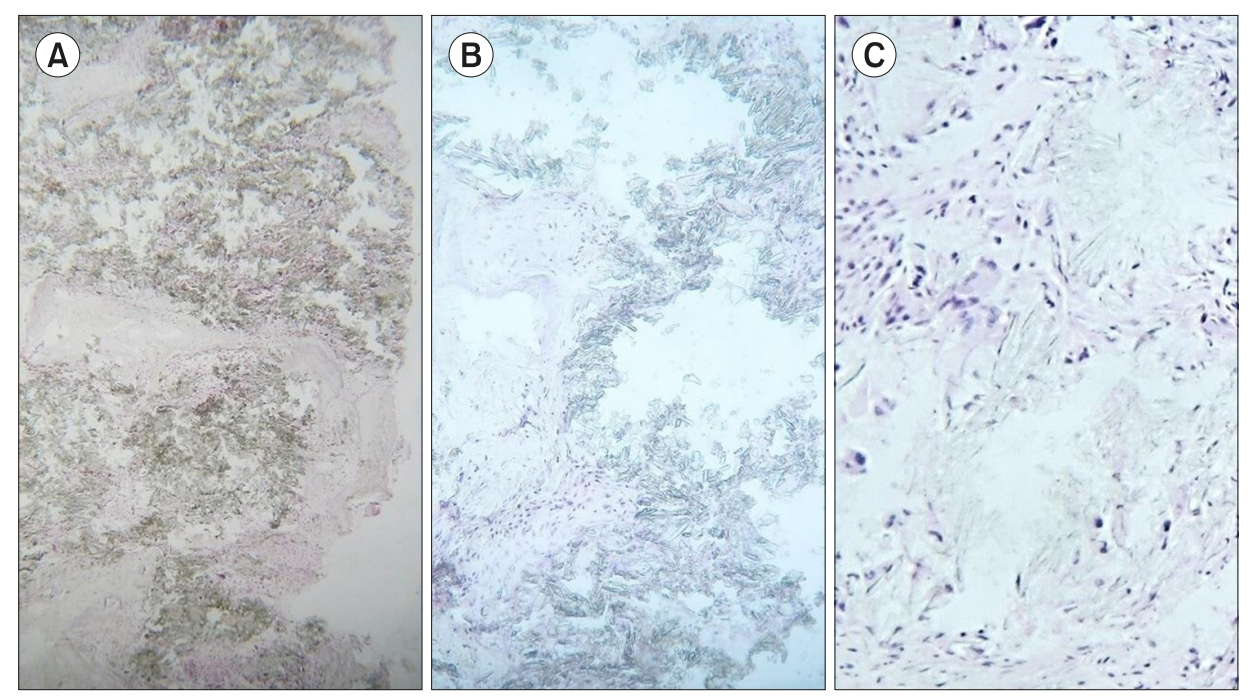

Systemic oxalosis is a rare autosomal recessive genetic metabolic disorder with an estimated prevalence of less than 1 case/million individuals/year. Alanine glyoxylate aminotransferase deficiency, leading to the endogenous overproduction of oxalic acid as well as the multisystem deposition of calcium oxalate crystals, including kidneys, cartilage, bone, soft tissues, liver, spleen, retina, peripheral nerves, and heart, whereas their bone marrow (BM) localization is very rarely observed. Chronic renal failure (CRF) and pancytopenia being the main clinical consequences. Herein, we describe the case of a 29-year-old woman with CRF on regular hemodialysis and transfusions. Her past medical history revealed primary hyperoxaluria. Blood count showed pancytopenia. Urinalysis revealed hyperoxaluria at $0.84 \mathrm{mmol} / \mathrm{L}$. BM histopathology revealed extensive deposition of large oxalate needle-like crystals arranged in a radial pattern within bony trabeculae (A), stromal fibrosis (B), striking foreign body giant cell reaction (C), thickened bony trabeculae (A, B), and absent residual hematopoiesis. Marked paratrabecular fibrosis was present trapping giant-cell reaction. In conclusion, Clinicians should be aware of the inclusion of oxalosis in the differential diagnosis of patients with unexplained cytopenia. In this clinical scenario, the histopathology of oxalosis is usually straightforward, confirming BM biopsy as an extremely useful tool for diagnosis. 